\title{
Wild-Animal Recognition in Agriculture Farms Using W-COHOG for Agro-Security
}

\author{
Nagaraju Andavarapu $^{1 *}$ and Valli Kumari Vatsavayi ${ }^{2}$ \\ ${ }^{1,2}$ Dept. of CS\&SE, Andhra University, Visakhapatnam, India.
}

\begin{abstract}
Computer Vision is applied in agriculture field for food grading, disease identification of the plants and agro-farms security. Huge crop damage is caused by the wild animal attacks on the agriculture farms. Here are some traditional techniques followed by the local farmers, but which are not effective. This problem can be solved using computer vision techniques. In this paper, we proposed an algorithm to detect animals in a given image. WCoHOG is a Histogram oriented gradients based feature vector with better accuracy. It is an extension of Co-occurrence Histograms of Oriented Gradients (CoHOG). In this paper LIBLINEAR classifier is used in order to get better accuracy for high dimensional data. The experiments were conducted on two benchmark datasets called Wild-Anim and CamaraTrap dataset. Experimental results prove that W-CoHOG performs better than existing state of the art methods
\end{abstract}

Keywords: Animal Detection, Wild Animal Detection, Agro Security, Weighted Co-occurrence Histograms of oriented Gradients, WCoHOG.

\section{INTRODUCTION}

Computer vision is applicable to many fields like medical field, robotics, remote sensing, machine vision, content-based image retrieval. Computer vision solves many problems in different disciplines. Computer vision also applied in the security field to perform automatic surveillance and access control and attendance management. The computer vision can be applied in agriculture field in many ways like disease detection of a tree by examining leaves or flowers or fruits and quality control of agricultural products. The computer vision techniques can be applied in order to 
provide security from wild animals in agriculture. In agriculture fields near to forest areas have a severe threat from wild animals, which attacks regularly on farms. These attacks causing huge damage to agricultural crops subsequently causes significant financial losses to farmers. According to a report prepared by Himachal Kisan Sabha reported that wild animals, including wild boars, monkeys, neelgai and etc, were causing a loss of Rs 400-500 Crore every year to farmers in Himachal Pradesh state, India[1]. Some measures are taken by the farmers by installing electrical fences to the farms, big flood lights in the form and other Jugaads [2] by the local farmers. Installing an electrical fence is much costlier to equip huge farms and kills so many animals, which is offensive legally and affects the biodiversity. Other existing techniques also are not effective due to several reasons. In this paper, we proposed a new and cost effective solution for agriculture security from wild animals. It is a proactive solution which gives alerts to the farmers when animals come near to the farms. In this paper, we proposed a solution that recognizes wild animals when it is captured on camera. In this paper, we use Weighted Co-occurrence histograms of oriented gradients (W-CoHOG) to recognize the animal in a given image.

The process is explained as below. Firstly images are captured from the cameras installed in the farm. Then, these images are processed to detect whether any animal exists in the given image. Later, if an animal present in animage, then recognition is done using W-CoHOG and this result will be sent to the farmer through the communication module.

\section{LITERATURE SURVEY}

Histogram based feature vectors are popular due to high detection rate and robustness. Histogram Oriented Gradients (HOG) [13] used in computer vision applications like object recognition, Content-based Image retrieval and people detection in videos. In HOG, Gradients of an image calculated and then converted into orientations. Later histograms calculated for these oriented gradients. There are different variants of HOG proposed by different people with improved accuracy CoHOG is an improved technique than HOG which uses a pair of oriented gradients to calculate cooccurrence matrix in order to improve the accuracy. W-CoHOG [5] is an extension of CoHOG [4], in this method magnitude component combined to a co- occurrence histogram matrix in order to improve the accuracy.

Wild Animal detection techniques [15] are proposed by different people earlier with different approaches. Varsha Bapat et al. proposed a system using wireless sensor networks to detect the animals in the field [12]. But sensor based solutions are costlier due to installing more sensors in the field increase the coverage and makes solution costlier.

Slavomir Matuska et al. proposed solution using computer vision technique, the 
method uses a combination of SIFT[7] and SURF[6] local features in order to detect the animals[3]. Shalika et. al. proposed animal classification system[14] using shape, colour and texture based feature vector and Support vector machine[19]. Mansi Parikh and Mrinal Patel proposed Animal detection using template matching algorithm [17] to prevent animal-vehicle accidents. Mriganka Gogoi and Savio Raj Philip [16] used segmentation technique for animal detection later SIFT[7] features used to recognize animals. Burghardt, Tilo, and Janko Calic implemented animal face detection and tracking system [18] using Harr Cascade classifier, Haar-like features [8] and AdaBoost classifiers [9]. Okafor, Emmanuel, et al. done acomparative study on wild animal recognition between deep learning and bag of visual words. Ronald kays et al. developed a system using motion-sensitive camera traps[20]. The images are captured by triggering cameras whenever motion detected by the cameras.

\section{COHOG}

Co-occurrence histogram oriented gradient technique widely used in object detection and recognition due to high accuracy and less false positive rates. CoHOG is an extension of histogram oriented gradients (HOG). In HOG, for a given image gradients are calculated for each image and converted into nine orientations. Finally, histograms are calculated for non-overlapping small regions of the image. In CoHOG, apair of oriented gradients is used instead of one orientation which improves accuracy. Co-occurrence matrix calculated for each pair of orientations of each small region of the image. Co-occurrence matrix calculated for different offsets for each region. The equation (1) shows the process of co-occurrence matrix for a given input image $I$. Figure 1 shows the overview of co-occurrence matrix and possible offsets for a given image region.

$$
C_{p, q}(x, y)=\sum_{i=1}^{n} \sum_{j=1}^{m}\left\{\begin{array}{cr}
1, \text { if } I(i, j)=x \text { and } I(i+p, j+q)=y \\
\text { Noner }
\end{array}\right.
$$

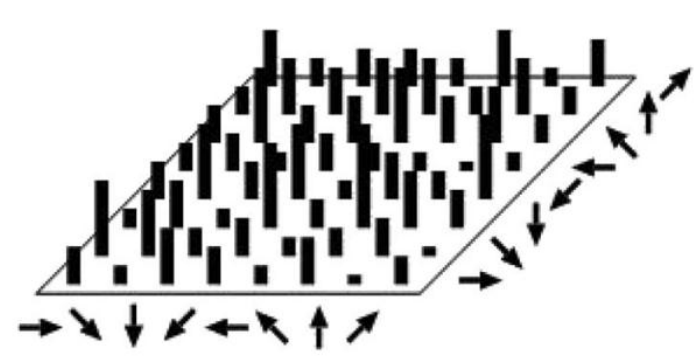

(a)

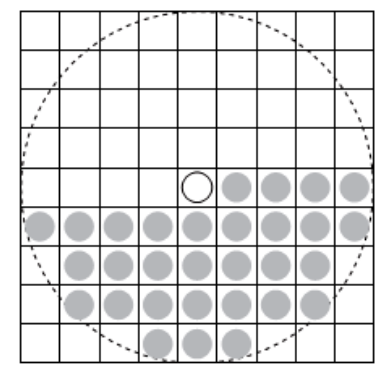

(b)

Figure 1: Co-occurrence matrix and offsets

a: Typical Co-occurrence matrix

b: Possible offsets for co-occurrence matrix 


\section{PROPOSED SYSTEM}

The proposed system uses W-CoHOG feature vector to implement animal recognition. The overview of thesystem as shownin figure 1. Firstly image is captured from the camerasat fixed intervals. The image is preprocessed in order to achievebetter accuracy. Later, theimage is processed with sliding window technique in order to identify the animal in the image. W-CoHOG feature vector used to calculate the feature vector. This feature vector supplied to the classifier to detect the animal in a particular window. In sliding window technique different sliding windows used in order to identify animals in different sizes of animal and zoom level of the camera.

In the CoHOG method, gradient directions are used to calculate feature vector and the magnitude is ignored. In the proposed method magnitude is also considered to extract more robust features. Weighted Co-occurrence Histograms of Oriented Gradients (W$\mathrm{CoHOG}$ ) is used for more robust feature descriptors than CoHOG. Figure (3) briefly explains the process of $\mathrm{W}-\mathrm{CoHOG}$ extraction method.

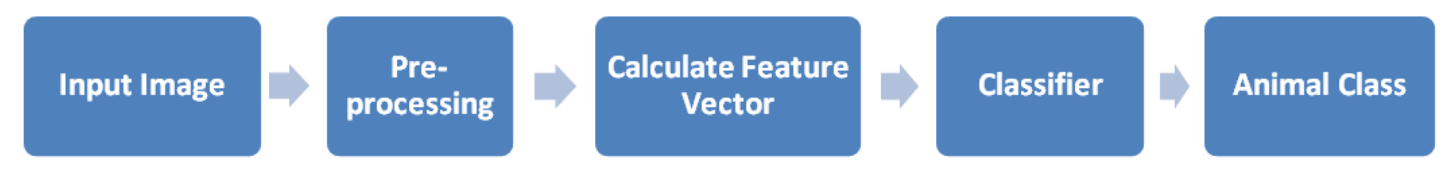

Figure 2: Overall Process of Proposed System

A given input image is pre-processed to enhance the image details. Later, gradients are calculated in magnitude and direction representation and converted into eight orientations. Next, the image is divided into $6 \times 6$ or $12 \times 12$ sized non-overlapping cells. Weighted co-occurrence matrices is calculated for each small cell. Finally, cooccurrence matrices of all cells are combined as asingle row.

\subsection{Pre-processing}

Pre-processing operations are performed at the lowest level of abstraction, which reduces the noise, distortions and enhances the image data or particular interest parts in the image. Firstly, Histogram equalization is performed on a given input image to enhance the contrast in the given image. Later sharpening filter is applied to the image to enhanceand highlight the region of interest and causes better object recognition.

\subsection{Feature Extraction}

After preprocessing the image, the feature vector calculation is performed using Weighted Co-occurrence Histogram Oriented Gradients technique. Firstly, gradients are computed for a given image in magnitude and direction form using popular operators like Sobel and Roberts operators. Equation (2),(3) shows operators of Sobel 
and Robert's filters respectively. These $\mathbf{g}_{x}$ and $\mathbf{g}_{\boldsymbol{y}}$ are calculated by convoluting two matrices on a given input image $A$.

\subsubsection{Sobel Operator}

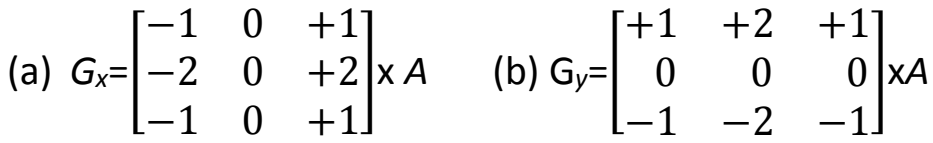

\subsubsection{Robert's Operator}
(a) $G_{x}=\left[\begin{array}{rr}+1 & 0 \\ 0 & -1\end{array}\right] \times A$
(b) $G_{y}=\left[\begin{array}{rr}0 & +1 \\ -1 & 0\end{array}\right] \times A$

(3)

Later, Gx and Gy are translated into gradient magnitude $(M)$ and direction $(\theta)$ form as shown in Equation (4). The gradient magnitude matrix $(M)$ is convoluted with $\mathrm{C}_{7 \times 7}$ shown inequation (5) which removes the aliasing effect. The gradient directions $(\theta)$ are converted into eight equal bins with $45^{\circ}$ intervals.

(a) $\theta=\tan ^{-1} \frac{g_{x}}{g_{y}}$ (b) $m=\sqrt{g_{x}^{2}+g_{y}^{2}}$

$$
C_{7 \times 7}=\frac{1}{49}\left[\begin{array}{lllllll}
1 & 1 & 1 & 1 & 1 & 1 & 1 \\
1 & 1 & 1 & 1 & 1 & 1 & 1 \\
1 & 1 & 1 & 1 & 1 & 1 & 1 \\
1 & 1 & 1 & 1 & 1 & 1 & 1 \\
1 & 1 & 1 & 1 & 1 & 1 & 1 \\
1 & 1 & 1 & 1 & 1 & 1 & 1 \\
1 & 1 & 1 & 1 & 1 & 1 & 1
\end{array}\right]
$$

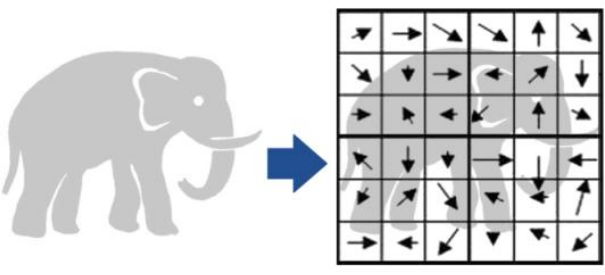

(a) Input Image

(b) Oriented Gradients

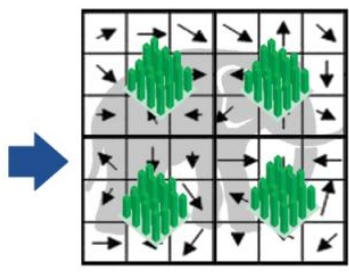

(C) Weighted Co-occurence

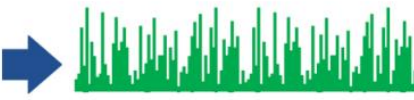

(d) Vectorization

$$
\text { Matrix }
$$

Figure 4: Overview of $\mathrm{W}-\mathrm{CHOG}$ calculation

\subsection{Weight Function}

In this proposed method, themagnitudes of the gradients are used in weight function inorderto compute weighted co-occurrence matrix. Firstly, the magnitudes of the input image are calculated followed by calculating magnitude weighted co-occurrence matrix calculation. Weight function is applied to the co-occurrence matrix to improve accuracy. The weight function is calculated for each small region of the image as shown below. 
Equation 6 and 7 explains the process of calculating Weighted Co-occurrence Matrix, where $\Delta x, \Delta y$ arethe offsets of the co-occurrence matrix.

$$
\begin{aligned}
C_{\Delta x, \Delta y}(i, \mathrm{j}) & =\sum_{p=1}^{n} \sum_{q=1}^{m}\left\{W_{(p, q),(p+\Delta x, p+\Delta y)} * \alpha\right. \\
\text { Where } \alpha & =\left\{\begin{array}{c}
1 \quad \text { ifo }(p, q)=\operatorname{iandO}(p+\Delta x, q+\Delta y)=j \\
0
\end{array} \quad\right. \text { Otherwise }
\end{aligned}
$$

In a given input image $\Delta x, \Delta y$ arethe offsets of the co-occurrence matrix. The weighted co-occurrence matrix is calculated for each small region in the image. The weight function $\mathrm{W}$ is calculated by using the magnitudeof the gradients of the image. Equation 8 shows the weight function for a given input image.

$$
W_{(p, q),(p+\Delta x, p+\Delta y)}=\left(\frac{m_{p, q}}{\bar{M}} * \frac{m_{p+\Delta x, p+\Delta y}}{\bar{M}}\right)+\mu
$$

Let $m_{p, q}$ is gradient magnitude of $\mathrm{p}$, q location in a given input image. $\bar{M}$ is mean of gradient magnitudes of an image I and $\mu$ is a constant and equals to one. Likewise, magnitude weighted co-occurrence matrix is computed for each small region of the image. The typical co-occurrence matrix is a $8 \times 8$ matrix because the gradients are divided into eight orientations. Finally, all weighted co-occurrence matrices are vectorized by concatenating all the matrices elements into a single row.Offsets of cooccurrence matrix playcrucial role in feature vector. Near offset represents local features of the object and far offsets represent global features.Among 31 possible offsets two offsets sufficient for animal recognition, and selected a pair which contains local feature and global features.

\subsection{Dimensionality Reduction}

Principal Component Analysis [24] is a dimensionality reduction technique which reduces the dimensionality of the feature vector. Dimensionality reduction also saves the memory to store the feature vector. The dataset consists of thousands of images of different classes which require larger memory to load the feature vector. Applying PCA reduces feature vector drastically without affecting the accuracy of the feature vector. The feature vector sizeof W-CoHOG is 4096 for two offset values reduced to 200 dimensions.

\subsection{Classification}

The feature vector size is very high in W-CoHOG due to histograms of co-occurrence matrix calculated for different offset. The proposed method contains higher dimensionality than sample size and multi-classes, where support vector machine performs well. LIBLINEAR [21] is an open source library for large-scale linear classifier and performs faster than SVM for huge instances of data. It supports different kernel functions and multi-class classification [22]. In this proposed method, multi-class non-linear SVM classifier uses LIBLINEAR. 


\section{EXPERIMENTAL RESULTS}

The experiments were conducted on two benchmark datasets CameraTrap dataset [20] and Wild-Anim dataset [10]. The Wild-Anim dataset contains 5000 images of five wild animals: bear, elephant, leopard, lion and wolf. The dataset is divided into five subsets which contain totally 1000 images for training and testing each subset. Each image in the dataset are $256 \times 256$ pixel images and further cropped to $128 \times 128$. The CameraTrap dataset contains 1000 images for each animal class. It contains twenty types of animals. But we merged to eight categories due to very similar features and names. For example, red-deer and roe-deer merged as deer class. We handpicked 400 images and cropped to $128 \times 128$ pixels and each class is further divided into two sets containing 300 and 100 for training and testing respectively. Figure (4) shows sample images from two datasets.
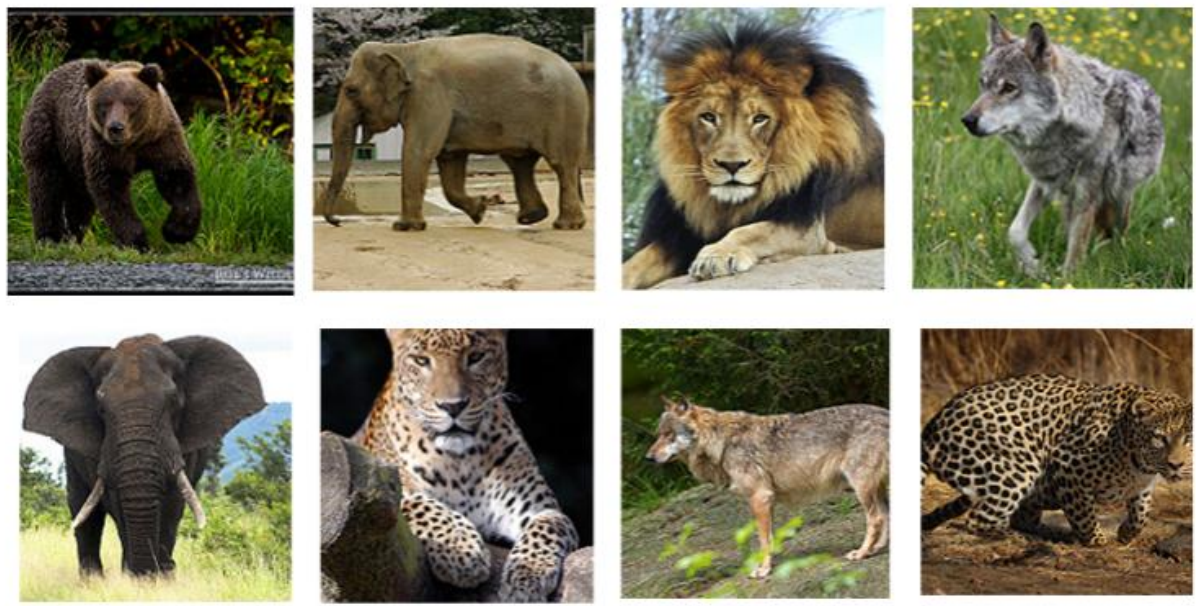

Figure 4: Sample Images of Wild-Anim Dataset
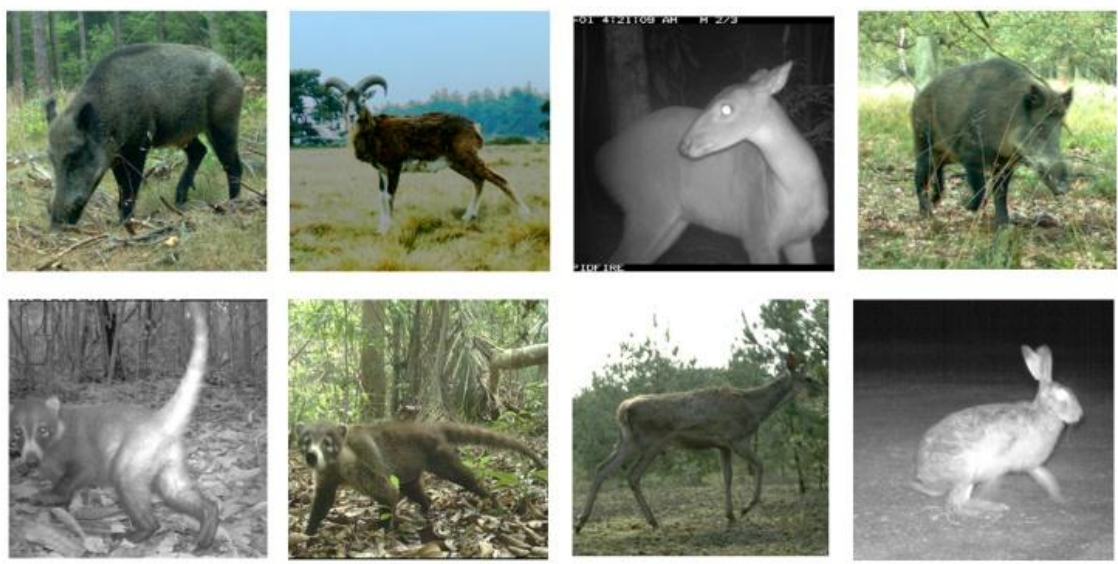

Figure 5: Sample Images of CameraTrap Dataset 
The experiments were conducted in OpenCV 2.4 with python. OpenCV [23] is an open source library for computer vision algorithms implemented in $\mathrm{C}$ and $\mathrm{C}++$. It has API support with many programming languages like Python, Java, C\#, Matlab and etc. The proposed algorithm was developed in python-OpenCV and LIBLINEAR classifier was used for classification. Figure 4 shows the confusion matrix for two benchmark datasets.

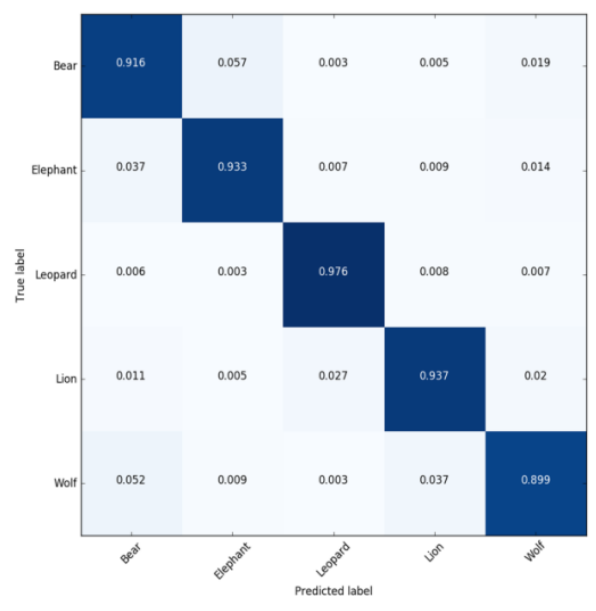

(a)
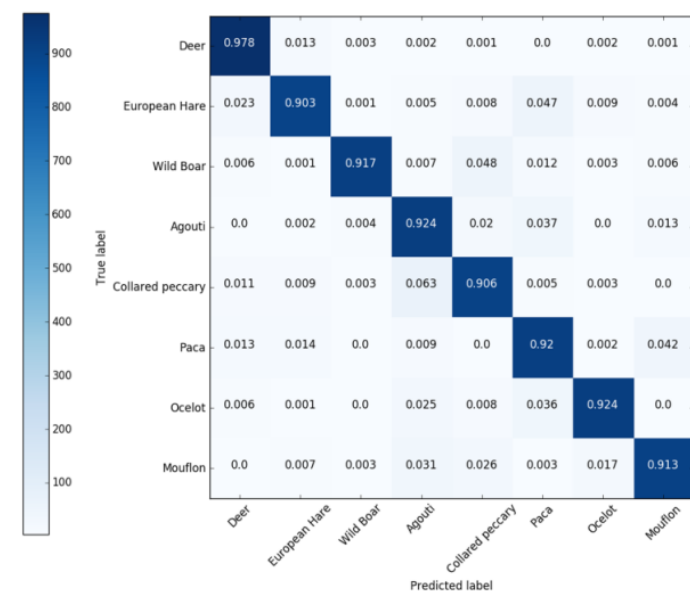

(b)

Figure 6: Confusion Matrix of Wild-Anim and CameraTrap Dataset a: WildAnim Dataset, b: CameraTrap Dataset

Table 1 Accuracy Comparison for Wild-Anim Dataset

\begin{tabular}{|l|c|c|c|c|}
\hline Object Class & HOG $(\%)$ & CoHOG $(\%)$ & R-CoHOG $(\%)$ & W-CoHOG(\%) \\
\hline Bear & 89.1 & 90.3 & 90.6 & 91.6 \\
\hline Elephant & 90.9 & 91.8 & 91.1 & 93.3 \\
\hline Leopard & 93.6 & 94.1 & 97.3 & 97.6 \\
\hline Lion & 91.4 & 92.3 & 93.5 & 93.7 \\
\hline Wolf & 87.2 & 87.8 & 89.1 & 89.9 \\
\hline
\end{tabular}

These results are further compared with the state of the art methods. The proposed algorithm shows better accuracy than existing techniques. Table (1) and (2) shows a comparison of the results with other popular algorithms. By observing the results our proposed method performs well on two benchmark datasets. 
Table 2 Accuracy Comparison for CameraTrap Dataset

\begin{tabular}{|l|c|c|c|c|}
\hline Object Class & HOG $(\%)$ & CoHOG $(\%)$ & R-CoHOG(\%) & W-CoHOG(\%) \\
\hline Deer & 94.5 & 94.9 & 97.6 & 97.8 \\
\hline European Hare & 89.9 & 90.1 & 90.4 & 90.3 \\
\hline Wild Boar & 88.7 & 89.9 & 90.3 & 91.7 \\
\hline agouti & 91.3 & 91.1 & 92.0 & 92.4 \\
\hline Collared peccary & 87.4 & 88.4 & 89.3 & 90.6 \\
\hline Paca & 90.3 & 90.8 & 91.1 & 92 \\
\hline Ocelot & 91.0 & 91.2 & 90.5 & 92.4 \\
\hline Mouflon & 89.4 & 90.5 & 91.0 & 91.3 \\
\hline
\end{tabular}

\section{CONCLUSION AND FUTURE WORK}

In this paper, we proposed a new algorithm for animal recognition. This method achieved better accuracy on two benchmark datasets compared to other existing algorithms. The experiments were conducted on static images. In future, we will implement a technique for recognizing animals in the video. In the future, we work on improving the algorithm to improve the accuracy.

\section{REFERENCES}

[1] 'News Report about wild animal attacks in agriculture field', http://www.tribuneindia.com/news/himachal/ community/wild- animalscausing-heavy-losses-to-farmers-in-state/94186.html, accessed 13 October 2016.

[2] 'Wikipedia',https://en.wikipedia.org/wiki/Jugaad,accessed 24 August 2016

[3] Matuska, Slavomir, et al.: 'Classification of wild animals based on SVM and local descriptors.' AASRI Procedia 9 (2014), pp. 25-30.

[4] Watanabe, Tomoki, Satoshi, Ito, and Kentaro Yokoi: 'Co-occurrence histograms of oriented gradients for pedestrian detection.' Pacific-Rim Symposium on Image and Video Technology. Springer Berlin Heidelberg, 2009.

[5] Andavarapu, Nagaraju, and Valli Kumari, Vatsavayi: 'Weighted CoHOG (WCoHOG) Feature Extraction for Human Detection.' Proceedings of Fifth International Conference on Soft Computing for Problem Solving. Springer Singapore, 2016. 
[6] Bay, Herbert, Tinne Tuytelaars, and Luc Van Gool: 'Surf: Speeded up robust features.' European conference on computer vision. Springer Berlin Heidelberg, 2006.

[7] Ng, Pauline C., and Steven Henikoff: 'SIFT: Predicting amino acid changes that affect protein function.' Nucleic acids research 31.13 (2003): 3812-3814.

[8] Lienhart, Rainer, and Jochen Maydt: 'An extended set of haar-like features for rapid object detection.' Image Processing. 2002. Proceedings. 2002 International Conference on. Vol. 1. IEEE, 2002.

[9] Niu, Zhiheng, et al.: '2d cascaded adaboost for eye localization.' Pattern Recognition, 2006. ICPR 2006. 18th International Conference on. Vol. 2. IEEE, 2006.

[10] Okafor, Emmanuel, et al.: 'Comparative Study Between Deep Learning and Bag of Visual Words for Wild-Animal Recognition.'

[11] Ho, Tin Kam 'Random Decision Forests.: ' Proceedings of the 3rd International Conference on Document Analysis and Recognition, Montreal, QC, 14-16 August 1995. pp. 278-282.

[12] Bapat, Varsha, et al.: 'WSN application for crop protection to divert animal intrusions in the agricultural land.' Computers and Electronics in Agriculture 133 (2017), pp. 88-96.

[13] Dalal, Navneet, and Bill, Triggs: 'Histograms of oriented gradients for human detection.' Computer Vision and Pattern Recognition, 2005. CVPR 2005. IEEE Computer Society Conference on. Vol. 1. IEEE, 2005.

[14] Shalika, AWD Udaya, and Lasantha, Seneviratne: 'Animal Classification System Based on Image Processing \& Support Vector Machine.' Journal of Computer and Communications 4.01 (2016), pp. 12.

[15] Sharma, Sachin, and D. J. Shah: 'A brief overview on different animal detection methods.' Signal \& Image Processing 4.3 (2013), pp. 77.

[16] Gogoi, Mriganka: 'PROTECTION OF CROPS FROM ANIMALS USING INTELLIGENT SURVEILLANCE SYSTEM.' Journal of Applied and Fundamental Sciences 1.2 (2015): 200.

[17] Parikh, Mansi, Miral Patel, and D., Bhatt: 'Animal detection using template matching algorithm.' International Journal of Research in Modern Engineering and Emerging Technology (IJRMEET) 1.3 (2013), pp. 26-32.

[18] Burghardt, Tilo, and Janko Calic.: 'Real-time face detection and tracking of animals.' Neural Network Applications in Electrical Engineering, 2006. NEUREL 2006. 8th Seminar on. IEEE, 2006. 
[19] Suykens, Johan AK, and Joos Vandewalle: 'Least squares support vector machine classifiers.' Neural processing letters 9.3 (1999), pp. 293-300.

[20] Kays, Roland, et al.: 'Monitoring wild animal communities with arrays of motion sensitive camera traps.' arXiv preprint arXiv:1009.5718 (2010).

[21] Fan, Rong-En, et al.: 'LIBLINEAR: A library for large linear classification.' Journal of machine learning research 9.Aug (2008), pp. 1871-1874.

[22] Crammer, Koby, and Yoram Singer.: 'On the algorithmic implementation of multiclass kernel-based vector machines.' Journal of machine learning research 2.Dec (2001), pp. 265-292.

[23] Bradski, Gary., and Adrian Kaehler.:'Learning OpenCV: Computer vision with the OpenCV library' (O'Reilly Media Inc., 2008).

[24] Jolliffe, Ian.:'Principal component analysis' (John Wiley \& Sons Ltd, 2002). 
